\title{
Translation invariance and related properties of $\mu$-pseudo almost automorphic (periodic) functions with application
}

\author{
Chuan-Yun Gu${ }^{1,2}$ and Hong-Xu Li ${ }^{* *}$ (iD
}

\section{"Correspondence:}

hoxuli@scu.edu.cn

'Department of Mathematics,

Sichuan University, Chengdu, P.R.

China

Full list of author information is

available at the end of the article

\section{Springer}

\begin{abstract}
It is well known that many important properties of $\mu$-pseudo almost automorphic (periodic) functions are based on the translation invariance. In this paper we give a new result of the translation invariance with conditions weaker than the known one. Then some more properties are studied, including the uniqueness of the decomposition and the convolution of the space. Moreover, we present a result on the equality of two spaces of this kind of functions, which extends the well-known results to the case when the measures of the spaces are not equivalent. As an application, we give an existence and uniqueness theorem of $\mu$-pseudo almost automorphic mild solution for a semilinear fractional differential equation.
\end{abstract}

MSC: $34 \mathrm{C} 27 ; 35 \mathrm{~B} 15$

Keywords: $\mu$-pseudo almost automorphic (periodic); Translation invariance; Convolution theorem; Measure theory; Fractional differential equation

\section{Introduction}

Almost periodicity and almost automorphy are attractive topics in the qualitative theory of differential equations due to their significance and applications in physics, mathematical biology, control theory and others. Then these concepts are generalized in various ways, say, pseudo almost periodicity (Zhang [1-3]), weighted pseudo almost periodicity (Diagana $[4,5])$, pseudo almost automorphy (Liang, Xiao and Zhang [6, 7]), weighted pseudo almost automorphy (Blot et al. [8]), etc. These concepts have been widely used in the investigation of ordinary differential equations, partial differential equations, functional differential equations and fractional differential equations. As a result, a vast of contributions were generated (see e.g. [9-18] and the references therein).

Recently, by using the measure theory, Blot, Cieutat and Ezzinbi $[19,20]$ introduced the concepts of $\mu$-pseudo almost periodicity and $\mu$-pseudo almost automorphy which are generalization of weighted pseudo almost periodicity and weighted pseudo almost automorphy, respectively. Some basic properties with applications are presented.

We notice that many applications of $\mu$-pseudo almost automorphic (periodic) functions (abbr. $\mu$-paa ( $\mu$-pap) functions) are based on the uniqueness of their decompositions, and this relies on the translation invariance of this kind of functions. So we give a new result

(c) The Author(s) 2018. This article is distributed under the terms of the Creative Commons Attribution 4.0 International License (http://creativecommons.org/licenses/by/4.0/), which permits unrestricted use, distribution, and reproduction in any medium, provided you give appropriate credit to the original author(s) and the source, provide a link to the Creative Commons license, and indicate if changes were made. 
of the translation invariance, and then some more properties are studied, including the uniqueness of the decomposition and the convolution of the spaces.

Meanwhile, it known that two spaces of $\mu$-paa ( $\mu$-pap) functions are the same if the measures of the spaces are equivalent. But it is unclear that whether two spaces are the same or not if their measures are not equivalent. In this paper, we present some sufficient conditions for the equality of the spaces including the case when the measures are not equivalent. Our results improve some well-known results (see Remarks 3.3, 3.8, 3.11 and Examples 3.4, 3.12).

We note that, for the case of weighted pseudo almost automorphic (periodic) functions (abbr. wpaa (wpap) functions), there were several important results on the topic of this paper. The completeness of wpaa space was firstly proved in [21] without any restriction on the weight, the translation invariance has been studied in [22-24], and a result on the equivalent of the wpaa (wpap) spaces with nonequivalent weights was obtained in [24]. Moreover, some basic properties of wpap sequences were studied in [25]. We note also that the completeness of $\mu$-paa ( $\mu$-pap) space was obtained in [26] by proving some abstract results on the characterization of the closedness of the sum of two closed vector subspaces of a Banach space.

At last, as an application, we give an existence and uniqueness theorem of $\mu$-paa mild solution for a semilinear fractional differential equation. Some examples are given to illustrate the abstract results.

\section{Preliminaries}

We first introduce some classical notations. Let $(\mathbb{X},\|\cdot\|),(\mathbb{Y},\|\cdot\|)$ be two Banach spaces, and $B C(\mathbb{R}, \mathbb{X})$ (resp. $B C(\mathbb{R} \times \mathbb{Y}, \mathbb{X}))$ be the space of bounded continuous functions $u$ : $\mathbb{R} \rightarrow \mathbb{X}($ resp. $u: \mathbb{R} \times \mathbb{Y} \rightarrow \mathbb{X})$. Endowed with the sup norm $\|u\|=\sup _{t \in \mathbb{R}}\|u(t)\|, B C(\mathbb{R}, \mathbb{X})$ is a Banach space. We note that even though the notation $\|\cdot\|$ is used for norms in different spaces, no confusion should arise.

Definition 2.1 ([2]) A continuous function $f: \mathbb{R} \rightarrow \mathbb{X}$ is called almost periodic if for each $\varepsilon>0$ there exists $l(\varepsilon)>0$ such that every interval of length $l(\varepsilon)$ contains a number $\tau$ with the property that $\|f(t+\tau)-f(t)\|<\varepsilon$ for each $t \in \mathbb{R}$. Denote by $A P(\mathbb{X})$ the set of all such functions.

Definition 2.2 ([27]) A continuous function $f: \mathbb{R} \rightarrow \mathbb{X}$ is called almost automorphic if for every sequence of real numbers $\left\{s_{n}^{\prime}\right\}$, there exists a subsequence $\left\{s_{n}\right\}$ such that $g(t)=$ $\lim _{n \rightarrow+\infty} f\left(t+s_{n}\right)$ is well defined for $t \in \mathbb{R}$, and $\lim _{n \rightarrow+\infty} g\left(t-s_{n}\right)=f(t)$ for $t \in \mathbb{R}$. Denote by $A A(\mathbb{X})$ the set of all such functions.

Let $U$ be the set of all functions $\rho: \mathbb{R} \rightarrow(0,+\infty)$ which are locally integrable over $\mathbb{R}$. For $T>0$ and $\rho \in U$, set

$$
m(T, \rho)=\int_{-T}^{T} \rho(t) d t
$$

Define

$$
U_{\infty}=\left\{\rho \in U: \lim _{T \rightarrow+\infty} m(T, \rho)=+\infty\right\} .
$$


For $\rho \in U_{\infty}, T>0$ and $f \in B C(\mathbb{R}, \mathbb{X})$, denote

$$
\mathcal{W}(T, f, \rho)=\frac{1}{m(T, \rho)} \int_{-T}^{T}\|f(t)\| \rho(t) d t
$$

Then the weighted ergodic space $M_{0}(\mathbb{X}, \rho)$ is defined by

$$
M_{0}(\mathbb{X}, \rho):=\left\{f \in B C(\mathbb{R}, \mathbb{X}): \lim _{T \rightarrow+\infty} \mathcal{W}(T, f, \rho)=0\right\},
$$

the space $\operatorname{WPAP}(\mathbb{X}, \rho)$ of weighted pseudo almost periodic functions (abbr. $\rho$-pap functions) were introduced in $[4,5]$ :

$$
W P A P(\mathbb{X}, \rho)=\left\{f=g+\phi \in B C(\mathbb{R}, \mathbb{X}): g \in A P(\mathbb{X}), \phi \in M_{0}(\mathbb{X}, \rho)\right\}
$$

and the space WPAA $(\mathbb{X}, \rho)$ of weighted pseudo almost automorphic functions (abbr. $\rho$-paa functions) were introduced in [8]:

$$
W P A A(\mathbb{X}, \rho)=\left\{f=g+\phi \in B C(\mathbb{R}, \mathbb{X}): g \in A A(\mathbb{X}), \phi \in M_{0}(\mathbb{X}, \rho)\right\}
$$

If $\rho=1$, a $\rho$-pap ( $\rho$-paa) function is a classic pseudo almost periodic (pseudo almost automorphic) function (see $[2,3])$.

Throughout this work, we denote by $\mathfrak{B}$ the Lebesgue $\sigma$-field of $\mathbb{R}$ and by $\mathcal{M}$ the set of all positive measures $\mu$ on $\mathfrak{B}$ satisfying $\mu(\mathbb{R})=+\infty$ and $\mu([a, b])<+\infty$ for all $a, b \in \mathbb{R}(a<b)$.

Definition 2.3 $([19,20])$ Let $\mu \in \mathcal{M}$. A bounded continuous function $f: \mathbb{R} \rightarrow \mathbb{X}$ is said to be $\mu$-ergodic if

$$
\lim _{T \rightarrow+\infty} \frac{1}{\mu([-T, T])} \int_{[-T, T]}\|f(t)\| d \mu=0 .
$$

We denote the space of all such functions by $\varepsilon(\mathbb{X}, \mu)$. The spaces $P A P(\mathbb{X}, \mu)$ of $\mu$-pseudo almost periodic functions (abbr. $\mu$-pap functions) is given by

$$
P A P(\mathbb{X}, \mu)=\{f=g+\phi \in B C(\mathbb{R}, \mathbb{X}): g \in A P(\mathbb{X}), \phi \in \varepsilon(\mathbb{X}, \mu)\},
$$

and the spaces $P A A(\mathbb{X}, \mu)$ of $\mu$-pseudo almost automorphic functions (abbr. $\mu$-paa functions) is given by

$$
P A A(\mathbb{X}, \mu)=\{f=g+\phi \in B C(\mathbb{R}, \mathbb{X}): g \in A A(\mathbb{X}), \phi \in \varepsilon(\mathbb{X}, \mu)\} .
$$

Remark 2.4 From $[19,20]$, we have the following facts:

(i) Let $\rho$ be a nonnegative $\mathfrak{B}$-measurable function, and $\mu$ the positive measure defined by

$$
\mu(A)=\int_{A} \rho(t) d t \quad \text { for } A \in \mathfrak{B},
$$

where the integral is under the Lebesgue measure on $\mathbb{R}$. The function $\rho$ in (2.1) is called the Radon-Nikodym derivative of $\mu$ with respect to the Lebesgue measure 
on $\mathbb{R}$, and this is denoted by $\frac{d \mu}{d t}=\rho(t)$. In this case $\mu \in \mathcal{M}$ if and only if its Radon-Nikodym derivative $\rho$ is locally Lebesgue-integrable on $\mathbb{R}$ with

$$
\int_{-\infty}^{+\infty} \rho(t) d t=+\infty
$$

(ii) One can observe that a $\rho$-paa ( $\rho$-pap) function is $\mu$-paa ( $\mu$-pap) with $\rho(t)$ the Radon-Nikodym derivative of $\mu: \frac{d \mu}{d t}=\rho(t)$. Especially, a pseudo almost automorphic (pseudo almost periodic) function is a $\mu$-paa ( $\mu$-pap) function with $\mu$ the Lebesgue measure.

\section{Main results}

\subsection{Translation invariance of $P A A(\mathbb{X}, \mu)(P A P(\mathbb{X}, \mu))$}

Let $\tau \in \mathbb{R}$ and $f \in B C(\mathbb{R}, \mathbb{X})$. We denote by $f_{\tau}$ the translation function of $f$ defined by $f_{\tau}(s)=$ $f(\tau+s)$ for $s \in \mathbb{R}$. A subset $\mathcal{F}$ of $B C(\mathbb{R}, \mathbb{X})$ is said to be translation invariant if for all $f \in \mathcal{F}$ we have $f_{\tau} \in \mathcal{F}, \tau \in \mathbb{R}$. For $\mu \in \mathcal{M}$ and $\tau \in \mathbb{R}$, we denote $\mu_{\tau}$ the positive measure on $\mathfrak{B}$ defined by

$$
\mu_{\tau}(A)=\mu(\{a+\tau: a \in A\}) \quad \text { for } A \in \mathfrak{B} .
$$

For a set $A \subset \mathbb{R}$ and $\tau \in \mathbb{R}$, we denote $A^{\tau}=\{x+\tau: x \in A\}$ and $A_{T}^{\tau}=\{x+\tau: x \in[-T, T] \cap A\}$. For $\mu \in \mathcal{M}$, the following assumption will be needed later:

$\left(\mathrm{H}_{1}\right)$ For each $\tau \in \mathbb{R}$, there exist a constant $\beta>0$ and a measurable set $\Omega \subset \mathbb{R}$ such that

$$
\mu_{\tau}(A) \leq \beta \mu(A) \quad \text { for } A \in \mathfrak{B}, A \cap \Omega=\emptyset
$$

and

$$
\lim _{T \rightarrow+\infty} \frac{\mu\left(\Omega_{T}^{\tau}\right)}{\mu([-T, T])}=0
$$

Lemma 3.1 Let $\mu \in \mathcal{M}$ satisfying $\left(\mathrm{H}_{1}\right)$. Then, for $\tau \in \mathbb{R}$,

$$
\limsup _{T \rightarrow+\infty} \frac{\mu([-T-\tau, T+\tau])}{\mu([-T, T])}<+\infty
$$

Proof Let $\tau \in \mathbb{R}$. By $\left(\mathrm{H}_{1}\right)$, there exist constants $\beta_{1}, \beta_{2}>0$ and measurable sets $\Omega, \Theta \subset \mathbb{R}$ such that

$$
\begin{aligned}
& \mu_{\tau}(A) \leq \beta_{1} \mu(A) \quad \text { for } A \in \mathfrak{B}, A \cap \Omega=\emptyset, \\
& \mu_{-\tau}(A) \leq \beta_{2} \mu(A) \quad \text { for } A \in \mathfrak{B}, A \cap \Theta=\emptyset .
\end{aligned}
$$

Then

$$
\begin{aligned}
\mu_{\tau}([-T, T]) & =\mu_{\tau}([-T, T] \backslash \Omega)+\mu_{\tau}([-T, T] \cap \Omega) \\
& \leq \beta_{1} \mu([-T, T] \backslash \Omega)+\mu_{\tau}([-T, T] \cap \Omega) \\
& \leq \beta_{1} \mu([-T, T])+\mu\left(\Omega_{T}^{\tau}\right) .
\end{aligned}
$$


Similarly, we can get

$$
\mu_{-\tau}([-T, T]) \leq \beta_{2} \mu([-T, T])+\mu\left(\Theta_{T}^{-\tau}\right) .
$$

Let $T>|\tau|$, then we have

$$
\begin{aligned}
\mu([-T-\tau, T+\tau]) & \leq \mu([-T-\tau, T-\tau])+\mu([-T+\tau, T+\tau]) \\
& =\mu_{-\tau}([-T, T])+\mu_{\tau}([-T, T]) \\
& \leq\left(\beta_{1}+\beta_{2}\right) \mu([-T, T])+\mu\left(\Omega_{T}^{\tau}\right)+\mu\left(\Theta_{T}^{-\tau}\right) .
\end{aligned}
$$

This together with $\left(\mathrm{H}_{1}\right)$ yields

$$
\limsup _{T \rightarrow+\infty} \frac{\mu([-T-\tau, T+\tau])}{\mu([-T, T])} \leq \beta_{1}+\beta_{2}<+\infty .
$$

Theorem 3.2 Let $\mu \in \mathcal{M}$ satisfying $\left(\mathrm{H}_{1}\right)$. Then $\varepsilon(\mathbb{X}, \mu)$ is translation invariant. Consequently, $P A A(\mathbb{X}, \mu)$ and $P A P(\mathbb{X}, \mu)$ are translation invariant.

Proof Let $f \in \varepsilon(\mathbb{X}, \mu)$ and $\tau \in \mathbb{R}$. By the definition of $\mu$, there exists $T_{0}>0$ such that $\mu([-T-|\tau|, T+|\tau|])>0$ for $T>T_{0}$. Let $\beta$ and $\Omega$ be the ones given in $\left(\mathrm{H}_{1}\right)$ for $-\tau$. Then for $T>T_{0}$

$$
\begin{aligned}
\int_{[-T, T]}\left\|f_{\tau}(t)\right\| d \mu & =\int_{[-T, T]}\|f(t+\tau)\| d \mu \\
& =\int_{[-T, T] \backslash \Omega^{-\tau}}\|f(t+\tau)\| d \mu+\int_{[-T, T] \cap \Omega^{-\tau}}\|f(t+\tau)\| d \mu \\
& \leq \int_{[-T+\tau, T+\tau] \backslash \Omega}\|f(t)\| d \mu_{-\tau}+\|f\| \mu\left([-T, T] \cap \Omega^{-\tau}\right) \\
& \leq \beta \int_{[-T+\tau, T+\tau] \backslash \Omega}\|f(t)\| d \mu+\|f\| \mu\left(\Omega_{T+|\tau|}^{-\tau}\right) \\
& \leq \beta \int_{[-T-|\tau|, T+|\tau|]}\|f(t)\| d \mu+\|f\| \mu\left(\Omega_{T+|\tau|}^{-\tau}\right) .
\end{aligned}
$$

Now by $\left(\mathrm{H}_{1}\right)$, Lemma 3.1 and the fact that $f \in \varepsilon(\mathbb{X}, \mu)$,

$$
\begin{aligned}
& \frac{1}{\mu([-T, T])} \int_{[-T, T]}\left\|f_{\tau}(t)\right\| d \mu \\
& \quad=\frac{\mu([-T-|\tau|, T+|\tau|])}{\mu([-T, T])} \cdot \frac{1}{\mu([-T-|\tau|, T+|\tau|])} \int_{[-T, T]}\|f(t+\tau)\| d \mu \\
& \quad \leq \frac{\mu([-T-|\tau|, T+|\tau|])}{\mu([-T, T])} \cdot \frac{\beta \int_{[-T-|\tau|, T+|\tau|]}\|f(t)\| d \mu+\|f\| \mu\left(\Omega_{T+|\tau|}^{-\tau}\right)}{\mu([-T-|\tau|, T+|\tau|])} \\
& \quad \rightarrow 0 \quad \text { as } T \rightarrow+\infty .
\end{aligned}
$$

That is, $f_{\tau} \in \varepsilon(\mathbb{X}, \mu)$, and then $\varepsilon(\mathbb{X}, \mu)$ is translation invariant. Consequently $P A A(\mathbb{X}, \mu)$ and $P A P(\mathbb{X}, \mu)$ are translation invariant since $A P(\mathbb{X})$ and $A A(\mathbb{X})$ are. This completes the proof. 


\section{Remark 3.3}

(i) In $[19,20]$, the following assumption was used:

(H) For all $\tau \in \mathbb{R}$, there exist $\beta>0$ and a bounded interval $I$ such that

$$
\mu(\{a+\tau: a \in A\}) \leq \beta \mu(A), \quad \text { when } A \in \mathfrak{B} \text { satisfies } A \cap I=\emptyset .
$$

Notice that $(\mathrm{H})$ is named $\left(\mathrm{H}_{2}\right)$ in [20]. If $\Omega$ is a bounded interval, (3.1) holds automatically. Then $\left(\mathrm{H}_{1}\right)$ is weaker than $(\mathrm{H})$. As a result, Theorem 3.2 improves [19, Theorem 3.5] and [20, Theorem 3.3].

(ii) For the case of $\mu(A)=\int_{A} \rho(t) d t$, that is, of weighted pseudo almost automorphic (periodic) functions, the same conclusion of Theorem 3.2 was obtained in [24, Theorem 4.2, Remark 4.3] with a condition slight different from $\left(\mathrm{H}_{1}\right)$, and similar result was also given in [22, Theorem 3.7 (ii)] with different conditions.

The following example shows that some measure $\mu$ satisfies $\left(\mathrm{H}_{1}\right)$, but does not satisfy $(\mathrm{H})$.

Example 3.4 Let the Radon-Nikodym derivative $\rho$ of the measure $\mu$ be given as

$$
\rho(t)= \begin{cases}e^{-t}, & t \in(2 n-1,2 n], n \in \mathbb{N}_{+} \\ 1, & \text { otherwise }\end{cases}
$$

Then, for $\tau=1$, it is easy to get

$$
\mu_{1}((2 n-1,2 n])=1=\frac{e^{2 n}}{e-1} \mu((2 n-1,2 n]) .
$$

Notice that the interval $I$ in $(\mathrm{H})$ is bounded and $\frac{e^{2 n}}{e-1} \rightarrow+\infty$ as $n \rightarrow+\infty$. So $\mu$ does not satisfy $(\mathrm{H})$. However, it is easy to verify that $\mu$ satisfies $\left(\mathrm{H}_{1}\right)$ for $\beta=1$ and $\Omega=(1,+\infty)$.

\subsection{Related properties with respect to translation invariance}

Let us start with the construction of $\mu$-paa ( $\mu$-pap) functions through convolution. Let $\mathcal{L}(\mathbb{X})$ be the space of bounded linear maps from the Banach space $\mathbb{X}$ into itself, and $L^{1}(\mathbb{R}, \mathcal{L}(\mathbb{X}))$ the Lebesgue space with respect to the Lebesgue measure on $\mathbb{R}$. For $f \in$ $B C(\mathbb{R}, \mathbb{X})$ and $G \in L^{1}(\mathbb{R}, \mathcal{L}(\mathbb{X})), f * G$ is defined by

$$
(f * G)(t)=\int_{-\infty}^{+\infty} G(s) f(t-s) d s \quad \text { for } t \in \mathbb{R} .
$$

Clearly, $f * G \in B C(\mathbb{R}, \mathbb{X})$.

Theorem 3.5 Let $\mu \in \mathcal{M}$ satisfying $\left(\mathrm{H}_{1}\right)$, and $G \in L^{1}(\mathbb{R}, \mathcal{L}(\mathbb{X}))$. Assume that $f \in \varepsilon(\mathbb{X}, \mu)$, then $f * G \in \varepsilon(\mathbb{X}, \mu)$. Consequently, $f * G \in P A A(\mathbb{X}, \mu)(P A P(\mathbb{X}, \mu))$ if $f \in P A A(\mathbb{X}, \mu)$ $(\operatorname{PAP}(\mathbb{X}, \mu))$. 
Proof Let $f \in \varepsilon(\mathbb{X}, \mu)$. Notice that there exists $T_{0} \geq 0$ such that, for all $T \geq T_{0}$, $\mu([-T, T])>0$. Then, by Fubini's theorem, for $T \geq T_{0}$,

$$
\begin{aligned}
& \frac{1}{\mu([-T, T])} \int_{[-T, T]}\|(f * G)(t)\| d \mu \\
& \quad \leq \frac{1}{\mu([-T, T])} \int_{[-T, T]} \int_{-\infty}^{+\infty}\|G(s)\|\|f(t-s)\| d s d \mu \\
& \quad=\int_{-\infty}^{+\infty} \frac{\|G(s)\|}{\mu([-T, T])} \int_{[-T, T]}\|f(t-s)\| d \mu d s .
\end{aligned}
$$

By Theorem 3.2, we have $f(\cdot-s) \in \varepsilon(\mathbb{X}, \mu)$ for $s \in \mathbb{R}$. That is

$$
\lim _{T \rightarrow+\infty} \frac{1}{\mu([-T, T])} \int_{[-T, T]}\|f(t-s)\| d \mu=0 \quad \text { for } s \in \mathbb{R}
$$

Meanwhile,

$$
\frac{\|G(s)\|}{\mu([-T, T])} \int_{[-T, T]}\|f(t-s)\| d \mu \leq\|G(s)\|\|f\| \quad \text { for } s \in \mathbb{R} .
$$

Then it follows from the Lebesgue dominated convergence theorem that

$$
\begin{aligned}
& \lim _{T \rightarrow+\infty} \frac{1}{\mu([-T, T])} \int_{[-T, T]}\|(f * G)(t)\| d \mu \\
& \quad \leq \lim _{T \rightarrow+\infty} \int_{-\infty}^{+\infty} \frac{\|G(s)\|}{\mu([-T, T])} \int_{[-T, T]}\|f(t-s)\| d \mu d s=0 .
\end{aligned}
$$

That is $f * G \in \varepsilon(\mathbb{X}, \mu)$.

By the same arguments of the proofs in [19, Theorem 3.9] and [20, Theorem 3.8], we see that $f * G \in A A(\mathbb{X})(A P(\mathbb{X}))$ if $f \in A A(\mathbb{X})(A P(\mathbb{X}))$, and we omit the details here. As a result, we get the conclusion.

Furthermore, by Theorem 3.2 and similar arguments of the proofs of the corresponding results in $[19,20]$, we can obtain the following properties of $\mu$-paa ( $\mu$-pap) functions. Here we omit the details of the proofs.

Theorem 3.6 Let $\mu \in \mathcal{M}$ satisfying $\left(\mathrm{H}_{1}\right)$ and $f \in P A A(\mathbb{X}, \mu)(P A P(\mathbb{X}, \mu))$ be such that $f=$ $g+\phi$, where $g \in A A(\mathbb{X})(A P(\mathbb{X}))$ and $\phi \in \varepsilon(\mathbb{X}, \mu)$. Then $\{g(t): t \in \mathbb{R}\} \subset \overline{\{f(t): t \in \mathbb{R}\}}($ the closure of the range off).

Theorem 3.7 Let $\mu \in \mathcal{M}$ satisfying $\left(\mathrm{H}_{1}\right)$. The decomposition of a $\mu$-paa ( $\mu$-pap) function in the form $f=g+\phi$, where $g \in A A(\mathbb{X})(A P(\mathbb{X}))$ and $\phi \in \varepsilon(\mathbb{X}, \mu)$, is unique.

Remark 3.8 Theorems 3.5-3.7 improve the corresponding results in $[19,20]$ because $(H)$ implies $\left(\mathrm{H}_{1}\right)$ (see Remark 3.3). 


\subsection{Equality of two spaces of $\mu$-paa ( $\mu$-pap) functions}

Let $\mu_{1}, \mu_{2} \in \mathcal{M} . \mu_{1}$ is said to be equivalent to $\mu_{2}$ if there exist constants $\alpha$ and $\beta>0$ and a bounded interval $I$ such that

$$
\alpha \mu_{1}(A) \leq \mu_{2}(A) \leq \beta \mu_{1}(A) \text { for } A \in \mathfrak{B} \text { satisfying } A \cap I=\emptyset .
$$

From $[19,20]$, it is well known that the equivalence of $\mu_{1}$ and $\mu_{2}$ implies that $\varepsilon\left(\mathbb{X}, \mu_{1}\right)=$ $\varepsilon\left(\mathbb{X}, \mu_{2}\right)$, and consequently

$$
\operatorname{PAA}\left(\mathbb{X}, \mu_{1}\right)=P A A\left(\mathbb{X}, \mu_{2}\right) \quad \text { and } \quad P A P\left(\mathbb{X}, \mu_{1}\right)=\operatorname{PAP}\left(\mathbb{X}, \mu_{2}\right)
$$

However, it remains unclear whether we can get $\varepsilon\left(\mathbb{X}, \mu_{1}\right)=\varepsilon\left(\mathbb{X}, \mu_{2}\right)$ and (3.2) or not if $\mu_{1}$ and $\mu_{2}$ are not equivalent. The main purpose of this subsection is to deal with this problem.

Lemma 3.9 Let $\mu_{1}, \mu_{2} \in \mathcal{M}$. Assume that

$$
\limsup _{T \rightarrow+\infty} \frac{\mu_{1}([-T, T])}{\mu_{2}([-T, T])}<+\infty
$$

and there exist a measurable set $\Omega \subset \mathbb{R}$ and $\alpha>0$ such that

$$
\mu_{2}(A) \leq \alpha \mu_{1}(A) \quad \text { for } A \in \mathfrak{B} \text { satisfying } A \cap \Omega=\emptyset
$$

and

$$
\lim _{T \rightarrow+\infty} \frac{\mu_{2}([-T, T] \cap \Omega)}{\mu_{2}([-T, T])}=0 .
$$

Then $\varepsilon\left(\mathbb{X}, \mu_{1}\right) \subset \varepsilon\left(\mathbb{X}, \mu_{2}\right)$, and thus

$$
P A A\left(\mathbb{X}, \mu_{1}\right) \subset P A A\left(\mathbb{X}, \mu_{2}\right) \quad \text { and } \quad P A P\left(\mathbb{X}, \mu_{1}\right) \subset P A P\left(\mathbb{X}, \mu_{2}\right) \text {. }
$$

Proof Let $f \in \varepsilon\left(\mathbb{X}, \mu_{1}\right)$. Then, by (3.4),

$$
\begin{aligned}
& \frac{1}{\mu_{2}([-T, T])} \int_{[-T, T]}\|f(t)\| d \mu_{2} \\
& \quad=\frac{1}{\mu_{2}([-T, T])} \int_{[-T, T] \backslash \Omega}\|f(t)\| d \mu_{2}+\frac{1}{\mu_{2}([-T, T])} \int_{[-T, T] \cap \Omega}\|f(t)\| d \mu_{2} \\
& \quad \leq \alpha \cdot \frac{\mu_{1}([-T, T])}{\mu_{2}([-T, T])} \cdot \frac{1}{\mu_{1}([-T, T])} \int_{[-T, T] \backslash \Omega}\|f(t)\| d \mu_{1}+\frac{\mu_{2}([-T, T] \cap \Omega)}{\mu_{2}([-T, T])} \cdot\|f\| \\
& \quad \leq \alpha \cdot \frac{\mu_{1}([-T, T])}{\mu_{2}([-T, T])} \cdot \frac{1}{\mu_{1}([-T, T])} \int_{[-T, T]}\|f(t)\| d \mu_{1}+\frac{\mu_{2}([-T, T] \cap \Omega)}{\mu_{2}([-T, T])} \cdot\|f\| .
\end{aligned}
$$

This together with (3.3), (3.5) and the fact that $f \in \varepsilon\left(\mathbb{X}, \mu_{1}\right)$ implies

$$
\lim _{T \rightarrow+\infty} \frac{1}{\mu_{2}([-T, T])} \int_{[-T, T]}\|f(t)\| d \mu_{2}=0 .
$$

That is $f \in \varepsilon\left(\mathbb{X}, \mu_{2}\right)$, and then $\varepsilon\left(\mathbb{X}, \mu_{1}\right) \subset \varepsilon\left(\mathbb{X}, \mu_{2}\right)$. The proof is complete. 
Theorem 3.10 Let $\mu_{1}, \mu_{2} \in \mathcal{M}$. Assume that there exist constants $\alpha>0$ and $\beta>0$ and $a$ measurable set $\Omega \subset \mathbb{R}$ such that

$$
\alpha \mu_{2}(A) \leq \mu_{1}(A) \leq \beta \mu_{2}(A) \quad \text { for } A \in \mathfrak{B} \text { satisfying } A \cap \Omega=\emptyset
$$

and

$$
\lim _{T \rightarrow+\infty} \frac{\mu_{i}([-T, T] \cap \Omega)}{\mu_{i}([-T, T])}=0, \quad i=1,2 .
$$

Then $\varepsilon\left(\mathbb{X}, \mu_{1}\right)=\varepsilon\left(\mathbb{X}, \mu_{2}\right)$, and thus

$$
\operatorname{PAA}\left(\mathbb{X}, \mu_{1}\right)=P A A\left(\mathbb{X}, \mu_{2}\right) \quad \text { and } \operatorname{PAP}\left(\mathbb{X}, \mu_{1}\right)=\operatorname{PAP}\left(\mathbb{X}, \mu_{2}\right) .
$$

Proof By (3.6), (3.7) and Lemma 3.9, we need only to prove that (3.3) is true and

$$
\limsup _{T \rightarrow+\infty} \frac{\mu_{2}([-T, T])}{\mu_{1}([-T, T])}<+\infty .
$$

In fact, by (3.7), for $i=1,2$,

$$
\lim _{T \rightarrow+\infty} \frac{\mu_{i}([-T, T] \backslash \Omega)}{\mu_{i}([-T, T])}=\lim _{T \rightarrow+\infty} \frac{\mu_{i}([-T, T])-\mu_{i}([-T, T] \cap \Omega)}{\mu_{i}([-T, T])}=1 .
$$

Meanwhile, we have

$$
\frac{\mu_{1}([-T, T])}{\mu_{2}([-T, T])}=\frac{\mu_{2}([-T, T] \backslash \Omega)}{\mu_{2}([-T, T])} \cdot \frac{\mu_{1}([-T, T])}{\mu_{1}([-T, T] \backslash \Omega)} \cdot \frac{\mu_{1}([-T, T] \backslash \Omega)}{\mu_{2}([-T, T] \backslash \Omega)} .
$$

Then by (3.6)

$$
\begin{aligned}
\limsup _{T \rightarrow+\infty} \frac{\mu_{1}([-T, T])}{\mu_{2}([-T, T])} & =\limsup _{T \rightarrow+\infty} \frac{\mu_{1}([-T, T] \backslash \Omega)}{\mu_{2}([-T, T] \backslash \Omega)} \\
& \leq \limsup _{T \rightarrow+\infty} \frac{\beta \mu_{2}([-T, T] \backslash \Omega)}{\mu_{2}([-T, T] \backslash \Omega)}=\beta .
\end{aligned}
$$

That is (3.3) holds. Similarly, we can get (3.8). The proof is complete.

\section{Remark 3.11}

(i) We note that Theorem 3.10 improves [19, Theorem 2.20] and [20, Theorem 2.21]. Indeed, if the measurable set $\Omega$ is a bounded interval, i.e. $\mu_{1}$ and $\mu_{2}$ are equivalent, (3.7) holds automatically. Then Theorem 3.10 is the combination of [19, Theorem 2.20] and [20, Theorem 2.21].

(ii) We note that, for the case of $\mu(A)=\int_{A} \rho(t) d t$, that is, of weighted pseudo almost automorphic (periodic) functions, Theorem 3.10 is the same as [24, Theorem 2.1].

The following example shows the equality of the two spaces $P A A\left(\mathbb{X}, \mu_{1}\right)$ and $P A A\left(\mathbb{X}, \mu_{2}\right)$ $\left(P A P\left(\mathbb{X}, \mu_{1}\right)\right.$ and $\left.P A P\left(\mathbb{X}, \mu_{2}\right)\right)$ when $\mu_{1}$ and $\mu_{2}$ are not equivalent. 
Example 3.12 Let the Radon-Nikodym derivative $\rho_{1}, \rho_{2}$ of the measure $\mu_{1}, \mu_{2}$ be given as

$$
\rho_{1}(t)= \begin{cases}t^{-1}, & t \in(1,+\infty) \\ 1, & t \in(-\infty, 1]\end{cases}
$$

and

$$
\rho_{2}(t)= \begin{cases}e^{-t}, & t \in(0,+\infty) \\ 1, & t \in(-\infty, 0]\end{cases}
$$

Then

$$
\frac{\mu_{1}((n, n+1])}{\mu_{2}((n, n+1])}=\frac{\int_{(n, n+1]} \rho_{1}(t) d t}{\int_{(n, n+1]} \rho_{2}(t) d t}=\frac{\ln \left(1+\frac{1}{n}\right)}{e^{-n}-e^{-(n+1)}} \rightarrow+\infty \quad(n \rightarrow+\infty),
$$

and $\mu_{1}$ and $\mu_{2}$ are not equivalent. Thus [19, Theorem 2.20] and [20, Theorem 2.21] are not applicable. However, let $\Omega=(0,+\infty)$. It is easy to verify that all the assumptions of Theorem 3.10 hold. Then $\varepsilon\left(\mathbb{X}, \mu_{1}\right)=\varepsilon\left(\mathbb{X}, \mu_{2}\right)$, and thus (3.2) holds.

\section{$4 \mu$-paa mild solutions}

As an application of the results obtained in the last section, we study the existence and uniqueness of $\mu$-paa mild solutions of the following fractional differential equation:

$$
{ }^{C} D_{t}^{\alpha} u(t)=A u(t)+{ }^{C} D_{t}^{\alpha-1} f(t, u(t)), \quad t \in \mathbb{R}, 1<\alpha<2,
$$

where $A: D(A) \subset \mathbb{X} \rightarrow \mathbb{X}$ is a linear densely defined operator of sectorial type on a complex Banach space $\mathbb{X}$ and $f: \mathbb{R} \times \mathbb{X} \rightarrow \mathbb{X}$ is a $\mu$-paa function in $t$ for each $x \in \mathbb{X}$. The fractional derivative ${ }^{C} D_{t}^{\alpha}$ is to be understood in the Caputo sense.

Definition 4.1 ([28]) A closed linear operator $A$ with a dense domain $D(A)$ in a Banach space $\mathbb{X}$ is said to be sectorial of type $\omega$ with angle $\theta$ if there are constants $\omega \in \mathbb{R}, \theta \in\left(0, \frac{\pi}{2}\right)$, $M>0$ such that its resolvent exists outside the sector

$$
\omega+\Sigma_{\theta}:=\{\lambda+\omega: \lambda \in \mathcal{C},|\arg (-\lambda)|<\theta\}
$$

and

$$
\left\|(\lambda-A)^{-1}\right\| \leq \frac{M}{|\lambda-\omega|}, \quad \lambda \notin \omega+\Sigma_{\theta} .
$$

Definition 4.2 $([29,30])$ Let $1<\alpha<2$, and $A$ be a closed linear operator with a domain $D(A)$ in a Banach space $\mathbb{X}$. We say that $A$ is the generator of a solution operator if there exist $\omega \in \mathbb{R}$ and a strongly continuous function $S_{\alpha}: R_{+} \rightarrow \mathcal{L}(\mathbb{X})$ such that $\left\{\lambda^{\alpha}: \operatorname{Re} \lambda>\omega\right\} \subset \rho(A)$ and

$$
\lambda^{\alpha-1}\left(\lambda^{\alpha} I-A\right)^{-1} x=\int_{0}^{+\infty} e^{-\lambda t} S_{\alpha}(t) x d t, \quad \operatorname{Re} \lambda>\omega, x \in \mathbb{X}
$$

In this case, $S_{\alpha}(t)$ is called the solution operator generated by $A$. 
From [28], if $A$ is sectional of type $\omega \in \mathbb{R}$ with $0 \leq \theta<\pi\left(1-\frac{\alpha}{2}\right)$, then $A$ is a generator of a solution operator given by

$$
S_{\alpha}(t)=\frac{1}{2 \pi i} \int_{G} e^{\lambda t} \lambda^{\alpha-1}\left(\lambda^{\alpha}-A\right)^{-1} d \lambda, \quad t \geq 0,
$$

with $G$ a suitable path lying outside the sector $\omega+\Sigma_{\theta}$. Furthermore, the following lemma holds.

Lemma 4.3 ([28]) Let $A: D(A) \subset \mathbb{X} \rightarrow \mathbb{X}$ be a sectorial operator in a complex Banach space $\mathbb{X}$, satisfying hypotheses (4.2) and (4.3), for some $M>0, \omega<0$ and $0 \leq \theta<\pi\left(1-\frac{\alpha}{2}\right)$. Then there exists $C(\theta, \alpha)>0$ depending solely on $\theta$ and $\alpha$, such that

$$
\left\|S_{\alpha}(t)\right\|_{\mathcal{L}(\mathbb{X})} \leq \frac{C(\theta, \alpha) M}{1+|\omega| t^{\alpha}}, \quad t \geq 0
$$

Definition 4.4 ([31]) A continuous function $x: \mathbb{R} \rightarrow \mathbb{X}$ is called a mild solution of Eq. (4.1), if $s \rightarrow S_{\alpha}(t-s) f(s, x(s))$ is integrable on $(-\infty, t)$ for each $t \in \mathbb{R}$ and

$$
x(t)=\int_{-\infty}^{t} S_{\alpha}(t-s) f(s, x(s)) d s, \quad t \in \mathbb{R} .
$$

Definition 4.5 ([32]) A continuous function $f: \mathbb{R} \times \mathbb{X} \rightarrow \mathbb{Y}$ is said to be almost automorphic in $t$ uniformly with respect to $x$ in $\mathbb{X}$ if the following two conditions hold:

(i) for all $x \in \mathbb{X}, f(\cdot, x) \in A A(\mathbb{X})$,

(ii) $f$ is uniformly continuous on each compact set $\mathbb{K}$ in $\mathbb{X}$ with respect to the second variable $x$, namely, for $\epsilon>0$ and each compact set $\mathbb{K}$ in $\mathbb{X}$, there exists $\delta>0$ such that, for all $x_{1}, x_{2} \in \mathbb{K}$, one has

$$
\left.\left\|x_{1}-x_{2}\right\| \leq \delta \Rightarrow \sup _{t \in \mathbb{R}} \| f\left(t, x_{1}\right)-f\left(t, x_{2}\right)\right) \| \leq \epsilon
$$

Denote by $A A U(\mathbb{R} \times \mathbb{X}, \mathbb{Y})$ the set of all such functions.

Definition 4.6 $([19,20])$ Let $\mu \in \mathcal{M}$. A continuous function $f: \mathbb{R} \times \mathbb{X} \rightarrow \mathbb{Y}$ is said to be $\mu$-ergodic in $t$ uniformly with respect to $x$ in $\mathbb{X}$ if the following two conditions are true:

(i) for all $x \in \mathbb{X}, f(\cdot, x) \in \varepsilon(\mathbb{Y}, \mu)$,

(ii) $f$ is uniformly continuous on each compact set $\mathbb{K}$ in $\mathbb{X}$ with respect to the second variable $x$.

Denote by $\varepsilon U(\mathbb{R} \times \mathbb{X}, \mathbb{Y}, \mu)$ the set of all such functions.

Definition 4.7 ([19, 20]) Let $\mu \in \mathcal{M}$. A continuous function $f: \mathbb{R} \times \mathbb{X} \rightarrow \mathbb{Y}$ is said to be $\mu$ pseudo almost automorphic in $t$ uniformly with respect to $x$ in $\mathbb{X}$ if $f$ is written in the form $f=g+\phi$, where $g \in A A U(\mathbb{R} \times \mathbb{X}, \mathbb{Y}), \phi \in \varepsilon U(\mathbb{R} \times \mathbb{X}, \mathbb{Y}, \mu)$. Denote by $P A A U(\mathbb{R} \times \mathbb{X}, \mathbb{Y}, \mu)$ the set of all such functions.

Lemma 4.8 ([32]) Let $\mu \in \mathcal{M}, f \in P A A U(\mathbb{R} \times \mathbb{X}, \mathbb{Y}, \mu)$ and $x \in P A A(\mathbb{X}, \mu)$. Assume that the following hypothesis holds:

(C) For all bounded subset $\mathbb{B}$ of $\mathbb{X}, f(\mathbb{R} \times \mathbb{B})$ is bounded.

Then $f(\cdot, x(\cdot)) \in P A A(\mathbb{Y}, \mu)$. 
Lemma 4.9 Let $\mu \in \mathcal{M}$ satisfying $\left(\mathrm{H}_{1}\right)$ and $f \in P A A(\mathbb{X}, \mu)$. Assume that the integrable solution operator $S_{\alpha}(t)$ satisfies (4.4). Then the function $F$ defined by

$$
F(t):=\int_{-\infty}^{t} S_{\alpha}(t-s) f(s) d s, \quad t \in \mathbb{R}
$$

is in $P A A(\mathbb{X}, \mu)$.

Proof Let $G(t)=S_{\alpha}(t)$ if $t \geq 0$ and $G(t)=0$ if $t<0$. Then the conclusion follows from Theorem 3.5.

Theorem 4.10 Let $\mu \in \mathcal{M}$ satisfying $\left(\mathrm{H}_{1}\right), f \in P A A U(\mathbb{R} \times \mathbb{X}, \mathbb{X}, \mu)$ and let $A: D(A) \subset \mathbb{X} \rightarrow$ $\mathbb{X}$ be a sectorial operator in a complex Banach space $\mathbb{X}$, satisfying hypotheses (4.2) and (4.3), for some $M>0, \omega<0$ and $0 \leq \theta<\pi\left(1-\frac{\alpha}{2}\right)$. Assume that there exists $k>0$ such that

$$
\left\|f\left(t, x_{1}\right)-f\left(t, x_{2}\right)\right\| \leq k\left\|x_{1}-x_{2}\right\| \quad \text { for } t \in \mathbb{R}, x_{1}, x_{2} \in \mathbb{X},
$$

and

$$
k C(\theta, \alpha) M \frac{|\omega|^{-\frac{1}{\alpha}} \pi}{\alpha \sin \left(\frac{\pi}{\alpha}\right)}<1,
$$

where $C(\theta, \alpha)$ is the constant given in (4.4). Then Eq. (4.1) has a unique mild solution in $\operatorname{PAA}(\mathbb{X}, \mu)$.

Proof By (4.6), for $t \in \mathbb{R}, x \in \mathbb{X}$,

$$
\|f(t, x)\| \leq\|f(t, x)-f(t, 0)\|+\|f(t, 0)\| \leq k\|x\|+\|f(\cdot, 0)\| .
$$

Noticing that $\|f(\cdot, 0)\|<+\infty$ since $f(\cdot, 0) \in P A A(\mathbb{X}, \mu)$, then this means that, for a bounded set $\mathbb{B} \subset \mathbb{X}, f(\mathbb{R} \times \mathbb{B})$ is bounded. Let $x \in P A A(\mathbb{X}, \mu)$. Then $f(\cdot x(\cdot)) \in P A A(\mathbb{X}, \mu)$ by Lemma 4.8, and $\int_{-\infty}^{t} S_{\alpha}(t-\sigma) f(\sigma, x(\sigma)) d \sigma$ is $\mu$-paa by Lemma 4.9. Moreover, $P A A(\mathbb{X}, \mu)$ is a Banach space by [26, Theorem 4.11]. Now define an operator $\Phi: P A A(\mathbb{X}, \mu) \rightarrow$ $P A A(\mathbb{X}, \mu)$ by

$$
\Phi(x)(t):=\int_{-\infty}^{t} S_{\alpha}(t-\sigma) f(\sigma, x(\sigma)) d \sigma, \quad t \in \mathbb{R} .
$$

Let $x_{1}$ and $x_{2} \in P A A(\mathbb{X}, \mu)$, by Lemma 4.3 and (4.6),

$$
\begin{aligned}
\left\|\Phi\left(x_{1}\right)(t)-\Phi\left(x_{2}\right)(t)\right\| & \leq \int_{-\infty}^{t}\left\|S_{\alpha}(t-\sigma)\right\|\left\|f\left(\sigma, x_{1}(\sigma)\right)-f\left(\sigma, x_{2}(\sigma)\right)\right\| d \sigma \\
& \leq \int_{-\infty}^{t} \frac{C(\theta, \alpha) M}{1+|\omega|(t-\sigma)^{\alpha}} k\left\|x_{1}(\sigma)-x_{2}(\sigma)\right\| d \sigma \\
& \leq k\left\|x_{1}-x_{2}\right\| \int_{0}^{+\infty} \frac{C(\theta, \alpha) M}{1+|\omega| \sigma^{\alpha}} d \sigma
\end{aligned}
$$

for $t \in \mathbb{R}$. Notice also that

$$
\int_{0}^{+\infty} \frac{1}{1+|\omega| t^{\alpha}} d t=\frac{|\omega|^{-\frac{1}{\alpha}} \pi}{\alpha \sin \left(\frac{\pi}{\alpha}\right)} \quad \text { for } \alpha \in(1,2) .
$$


Then

$$
\left\|\Phi\left(x_{1}\right)-\Phi\left(x_{2}\right)\right\| \leq k C(\theta, \alpha) M \frac{|\omega|^{-\frac{1}{\alpha}} \pi}{\alpha \sin \left(\frac{\pi}{\alpha}\right)}\left\|x_{1}-x_{2}\right\| .
$$

This means that $\Phi$ is a contraction map by (4.7). It follows from the Banach contraction fixed point theorem, $\Phi$ has a unique fixed point $x \in P A A(\mathbb{X}, \mu)$, and $x$ is a $\mu$-paa mild solution of (4.1).

Example 4.11 To illustrate Theorem 4.10, we consider the following fractional relaxationoscillation equation:

$$
{ }^{C} D_{t}^{\alpha} u(t, x)=\frac{\partial^{2}}{\partial x^{2}} u(t, x)-a u(t, x)+{ }^{C} D_{t}^{\alpha-1} f(t, u(t, x)), \quad t \in \mathbb{R}, x \in[0, \pi],
$$

with boundary conditions $u(t, 0)=u(t, \pi)=0, t \in \mathbb{R}$, where $1<\alpha<2, a>0$ and

$$
f(t, u(t, x))=\sin \frac{1}{2+\cos t+\cos \pi t} \sin (u(t, x)), \quad t \in \mathbb{R} .
$$

Let the Radon-Nikodym derivative $\rho$ of the measure $\mu$ be defined by

$$
\rho(t)= \begin{cases}e^{-t}, & t \in(2 n-1,2 n], n \in \mathbb{N}_{+} \\ 1, & \text { otherwise }\end{cases}
$$

Set $\left(\mathbb{X},\|\cdot\|_{\mathbb{X}}\right)=\left(L^{2}([0, \pi]),\|\cdot\|_{2}\right)$. We assert that (4.8) has a unique solution in $\operatorname{PAA}(\mathbb{X}, \mu)$ if

$$
a>\left(\frac{C(\theta, \alpha) M \pi}{\alpha \sin \left(\frac{\pi}{\alpha}\right)}\right)^{\alpha}
$$

where $C(\theta, \alpha), M$ is as those in (4.4).

In fact, $\mu$ satisfies $\left(\mathrm{H}_{1}\right)$ by Example 3.4, and it is easy to see that $f \in P A A U(\mathbb{R} \times \mathbb{X}, \mathbb{X}, \mu)$. Define an operator $A$ on $\mathbb{X}$ by

$$
A \varphi=\varphi^{\prime \prime}-a \varphi, \quad \forall \varphi \in D(A)
$$

where $D(A):=\left\{\varphi \in \mathbb{X}: \varphi^{\prime \prime} \in \mathbb{X}, \varphi(0)=\varphi(\pi)=0\right\}$. It is well known that $\Delta u=u^{\prime \prime}$ is the infinitesimal generator of an analytic semigroup on $\mathbb{X}$. Thus $A$ is a sectorial of type $\omega=-a<0$. Now (4.8) can be formulated by (4.1), where $u(t)=u(t, \cdot)$. Meanwhile, we have

$$
\begin{aligned}
& \| f\left(t, u(t, \cdot)-f(t, v(t, \cdot)) \|_{2}\right. \\
& \quad \leq\left|\sin \frac{1}{2+\cos t+\cos \pi t}\right|\|\sin (u(t, \cdot))-\sin (v(t, \cdot))\|_{2} \\
& \quad \leq\|\sin (u(t, \cdot))-\sin (v(t, \cdot))\|_{2} \\
& \quad \leq\|u(t, \cdot)-v(t, \cdot)\|_{2}
\end{aligned}
$$


for all $u(t, \cdot), v(t, \cdot) \in L^{2}([0, \pi]), t \in \mathbb{R}$. This means that $f$ satisfies (4.6) with $k=1$. Notice that (4.9) implies (4.7). Therefore all the assumptions of Theorem 4.10 are satisfied, and then (4.8) has a unique solution in $P A A(\mathbb{X}, \mu)$.

\section{Acknowledgements}

The authors are grateful to the referees for the valuable comments and corrections. This work is supported by National Natural Science Foundation of China (Grant No. 11471227, 11561077) and Scientific Research Fund of Sichuan Provincial Education Department (No. 17ZB0370).

\section{Competing interests}

The authors declare that they have no competing interests.

\section{Authors' contributions}

All authors read and approved the final manuscript.

\section{Author details}

${ }^{1}$ Department of Mathematics, Sichuan University, Chengdu, P.R. China. ${ }^{2}$ Department of Mathematics, Sichuan University of Arts and Science, Dazhou, P.R. China.

\section{Publisher's Note}

Springer Nature remains neutral with regard to jurisdictional claims in published maps and institutional affiliations.

Received: 24 January 2018 Accepted: 2 May 2018 Published online: 08 May 2018

\section{References}

1. Zhang, C.: Integration of vector-valued pseudo-almost periodic functions. Proc. Am. Math. Soc. 121(1), 167-174 (1994)

2. Zhang, C.: Pseudo almost periodic solutions of some differential equations. J. Math. Anal. Appl. 181(1), 62-76 (1994)

3. Zhang, C.: Pseudo almost periodic solutions of some differential equations II. J. Math. Anal. Appl. 192(2), 543-561 (1995)

4. Diagana, T.: Weighted pseudo almost periodic functions and applications. C. R. Acad. Sci., Sér. 1 Math. 343(10), 643-646 (2006)

5. Diagana, T:: Weighted pseudo-almost periodic solutions to some differential equations. Nonlinear Anal. 68(8), 2250-2260 (2008)

6. Liang, J., Zhang, J., Xiao, T.J.: Composition of pseudo almost automorphic and asymptotically almost automorphic functions. J. Math. Anal. Appl. 340, 1493-1499 (2008)

7. Xiao, T.J., Liang, J., Zhang, J.: Pseudo almost automorphic solutions to semilinear differential equations in Banach space. Semigroup Forum 76, 518-524 (2008)

8. Blot, J., Mophou, G.M., N'Guérékata, G.M., Pennequin, D.: Weighted pseudo almost automorphic functions and applications to abstract differential equations. Nonlinear Anal. 71, 903-909 (2009)

9. Diagana, T.: Existence and uniqueness of pseudo-almost periodic solutions to some classes of partial evolution equations. Nonlinear Anal. 66(2), 384-395 (2007)

10. Liang, J., Xiao, T.J., Zhang, J.: Decomposition of weighted pseudo-almost periodic functions. Nonlinear Anal. 73 3456-3461 (2010)

11. Chang, Y.K., Zhang, R., N'Guérékata, G.M.: Weighted pseudo almost automorphic mild solutions to semilinear fractional differential equations. Comput. Math. Appl. 64, 3160-3170 (2012)

12. Ding, H.S., Liang, J., Xiao, T.J.: Almost automorphic solutions to nonautonomous semilinear evolution equations in Banach spaces. Nonlinear Anal. 73, 1426-1438 (2010)

13. Li, H.X., Zhang, L.L.: Stepanov-like pseudo-almost periodicity and semilinear differential equations with uniform continuity. Results Math. 59, 43-61 (2011)

14. Zhang, L.L., Li, H.X.: Weighted pseudo-almost periodic solutions for some abstract differential equations with uniform continuity. Bull. Aust. Math. Soc. 82, 424-436 (2010)

15. Mophou, G.M.: Weighted pseudo almost automorphic mild solutions to semilinear fractional differential equations. Appl. Math. Comput. 217, 7579-7587 (2011)

16. Xia, Z.N.: Weighted pseudo almost automorphic solutions of hyperbolic semilinear integro-differential equations. Nonlinear Anal. 9(5), 50-65 (2014)

17. Adimy, M., Ezzinbi, K., Marquet, C.: Ergodic and weighted pseudo-almost periodic solutions for partial functional differential equations in fading memory spaces. J. Appl. Math. Comput. 44, 147-165 (2014)

18. Ezzinbi, K., Miraoui, M.: $\mu$-pseudo almost periodic and automorphic solutions in the $\alpha$-norm for some partial functional differential equations. Numer. Funct. Anal. Optim. 36, 991-1012 (2015)

19. Blot, J., Cieutat, P., Ezzinbi, K.: Measure theory and pseudo almost automorphic functions: new developments and aplications. Nonlinear Anal. 75, 2426-2447 (2012)

20. Blot, J., Cieutat, P., Ezzinbi, K.: New approach for weighted pseudo almost periodic functions under the light of measure theory, basic results and applications. Appl. Anal. 92, 493-526 (2013)

21. Zheng, Z.M., Ding, H.S.: On completeness of the space of weighted pseudo almost automorphic functions. J. Funct. Anal. 268, 3211-3218 (2015)

22. Coronela, A., Pinto, M., Sepúlveda, D.: Weighted pseudo almost periodic functions, convolutions and abstract integral equations. J. Math. Anal. Appl. 435, 1382-1399 (2016) 
23. Ji, D., Zhang, C.: Translation invariance of weighted pseudo almost periodic functions and related problems. J. Math. Anal. Appl. 391, 350-362 (2012)

24. Ding, H.S., Liang, J., Xiao, T.J.: Weighted pseudo almost automorphic functions and WPAA solutions to semilinear evolution equations. J. Math. Anal. Appl. 409, 409-427 (2014)

25. Ding, H.S., N'Guérékata, G.M., Nieto, J.J.: Weighted pseudo almost periodic solutions for a class of discrete hematopoiesis model. Rev. Mat. Complut. 26, 427-443 (2013)

26. Blot, J., Cieutat, P.: Completeness of sums of subspaces of bounded functions and applications. Commun. Math. Anal. 19(2), 43-61 (2016)

27. Bochner, S.: A new approach to almost periodicity. Proc. Natl. Acad. Sci. USA 48, 2039-2043 (1962)

28. Cuesta, E.: Asymptotic bahaviour of the solutions of fractional integro-differential equations and some time discrtizations. Discrete Contin. Dyn. Syst. 2007(Supplement), 277-285 (2007)

29. Bazhlekova, E.G.: Fractional evolution equations in Banach spaces. Ph.D. thesis, Eindhoven University of Technology (2001)

30. Cuevas, C., Souza, J.C.: S-asymptotically $\omega$-periodic solutions of semilinear fractional integro-differential equations. Appl. Math. Lett. 22, 865-870 (2009)

31. Abbas, S., Kavitha, V., Murugesu, R.: Stepanov-like weighted pseudo almost automorphic solutions to fractional order abstract integro-differential equations. Proc. Indian Acad. Sci. Math. Sci. 125(3), 323-351 (2015)

32. Blot, J., Cieutat, P., N'Guérékata, G.M., Pennequin, D.: Superposition operators between various almost periodic function spaces and applications. Commun. Math. Anal. 6(1), 42-70 (2009)

\section{Submit your manuscript to a SpringerOpen ${ }^{0}$ journal and benefit from:}

- Convenient online submission

Rigorous peer review

- Open access: articles freely available online

- High visibility within the field

- Retaining the copyright to your article

Submit your next manuscript at $\gg$ springeropen.com 